CRITICA, Revisea Hispanoamericana de Fillosofta

Vol. XXII, Na. 64 (abril 1990): 41-53

\title{
NORMAS, SISTEMAS JURÍDICOS Y EFICACIA
}

\author{
Pablo Eugenio NavarRo \\ Consejo de Investigaciones Científicas y Tecnológicas \\ de la Provincia de Córdoba
}

\section{Introducción}

La explicación de las relaciones existentes entre las conductas debidas y las conductas producidas es un aspecto particularmente importante para el positivismo jurídico. ${ }^{1}$ Sin embargo, el problema de la eficacia es abordado, generalmente, en relación con otros temas tradicionales, por ejemplo: validez, condiciones de existencia de los sistemas jurídicos, etc. Dos cuestiones pueden señalarse como razones del escaso tratamiento independiente del tema de la eficacia:

(i) La eficacia de las normas es una cuestión dependiente de lo suscrito acerca del concepto de norma, sistema jurídico y acción humana. Por consiguiente, luego de resolver acerca de los criterios de individuación de normas y acciones, sólo resta analizar la coincidencia entre los contenidos normativos y los estados de cosas producidos por los sujetos normativos. Sin embargo, este argumento fracasa si el concepto de eficacia es utilizado para definir o identificar a las normas y sistemas jurídicos. Éste es el caso en Kelsen al postular las condiciones para suponer la Norma Básica Fundante, o en Hart al incluir la regla de reconocimiento en la clase de las reglas sociales, o en Ross al definir el concepto de "directiva", o en cualquier teoría que in-

${ }^{1}$ H. Kelsen, Teoria pura del derecho, UNAM, México, 1979, p. 219. 
serte a la eficacia como condición de existencia de los sistemas jurídicos.

(ii) La eficacia de las normas jurídicas es un problema de la sociología del derecho. Pero este punto de vista es erróneo, si distinguimos entre la eficacia como cuestión fáctica y la eficacia como cuestión conceptual. Ciertamente, la (in)eficacia de la ley de alquileres, o del código penal sancionado en 1921 son puntos correspondientes a la sociología jurídica. Pero definir bajo qué condiciones son eficaces las normas es una cuestión a establecer por la teoría general del derecho, de la misma manera en que ésta analiza el concepto de "aceptación" o de "validez".

En este trabajo mostraré los defectos y omisiones de algunas versiones habituales del concepto de eficacia y sugeriré una nueva manera de enfocar este problema. La conclusión que se desprende de mis argumentos - si es que son verdaderos-es que el concepto de eficacia plantea profundas interrogantes, y por lo tanto debemos rechazar una teoría que no dé cuenta de este problema.

\section{Eficacia y correspondencia}

Es fácil advertir que el término eficacia es ambiguo y que, según sea el significado escogido, las referencias de los enunciados de eficacia serán diferentes. Por ello, el interrogante a resolver no es si "eficacia" significa $C_{1}, C_{2} \ldots C_{n}$, sino cuál concepto de eficacia es apropiado para la teoría general del derecho. Obviamente, establecer los diferentes significados de eficacia es previo a escoger entre uno de ellos. La evaluación de la adecuación del concepto de eficacia puede efectuarse de acuerdo con los siguientes criterios: (a) vinculación entre el derecho como técnica de control social y las conductas de los sujetos; (b) incidencia real de las normas en las conductas de los sujetos. No profundizaré en la justificación de estos criterios, y solamente diré que ellos nos permiten vincular el pro- 
blema de la eficacia con las condiciones de existencia de los sitemas jurídicos, la especificidad de las funciones de los sistemas jurídicos, y -por último- ayudan a clarificar la posición que ocupa el concepto de eficacia en el discurso de la ciencia jurídica.

Supongamos una norma $(n)$ que pertenece al sistema normativo $S_{n}$ en el tiempo $t$; y también supongamos un sujeto $(s)$ que realiza el comportamiento prescrito por $(n)$. ¿Es suficiente la relación de correspondencia entre los estados de cosas y el contenido normativo para la eficacia de la norma $(n)$ ?

2a) La relación de correspondencia entre una acción y una prescripción es sólo un aspecto de la relación semántica entre un contenido proposicional y un estado de cosas. En el caso de la verdad de una proposición, lo que predicamos es la existencia de una correpondencia entre el significado de una sentencia y los hechos en el mundo. En el caso de las prescripciones, también podemos observar la correspondencia entre el contenido proposicional prescrito y las acciones o estados de cosas atribuibles a los sujetos. Pero mientras que es natural predicar eficacia de leyes prescriptivas, es una contradicción en los términos predicar ineficacia de una ley de la naturaleza. Explicar el concepto de eficacia solamente en términos de correspondencia no pone de manifiesto que el predicado "eficacia" es utilizado naturalmente en el caso de aquellos enunciados destinados a promover conductas, es decir, prescripciones. Por otra parte, aunque la relación de correspondencia es compatible con enunciados descriptivos, es un exceso de lenguaje calificar de eficaz a este tipo de enunciados. Esto muestra que el concepto de eficacia tiene que explicarse de manera tal de vincularlo específicamente con el concepto y función de las prescripciones. Incidentalmente, esto también muestra que no tiene sentido predicar eficacia en el caso de las normas permisivas. La característica específica de la eficacia no es la existencia de una relación de correspondencia sino el carácter 
deóntico del operador del contenido proposicional que se utiliza en los enunciados de eficacia. Esto se muestra claramente al considerar dos normas, una que permite el contenido proposicional $p$ y otra que obliga a producir $p$. Supongamos que los sujetos normativos hacen - $p$. Sólo en el segundo caso es natural predicar ineficacia, aun cuando sea verdadero también del primer caso que no existe relación de correspondencia entre las acciones de los sujetos y los contenidos normativos. Esto no significa que las normas permisivas sean irrelevantes, ya que éstas son una importante herramienta de análisis de la dinámica de los sistemas jurídicos. ${ }^{2}$

Si la eficacia se predica de una manera específica de caracterizar conductas (prescripciones), ello se debe solamente a que la función de las prescripciones es promover o evitar ciertas conductas. Por consiguiente, es un error desvincular el concepto de eficacia de los sistemas normativos de la función motivadora que caracteriza a estos sistemas. La relación genérica entre eficacia y motivación no sólo involucra al enunciado fáctico de la correspondencia, sino también un componente contrafáctico acerca de los cambios y acciones para el caso en que no hubiese existido el sistema normativo. Si, dado un sistema normativo $S_{n}$, los sujetos normativos produjeran los estados de cosas $p, q \ldots n$, y si no existiese $S_{n}$ los sujetos normativos igualmente ejecutaran $p, q \ldots n$ : ¿diríamos que el sistema es eficaz en cuanto técnica de promoción o control social? Hay que distinguir dos casos:

a) El nivel de conocimiento de los sujetos no incluye a $S_{n}$. La respuesta es negativa. La correspondencia entre $S_{n}$ y las acciones de los sujetos es, en el mejor de los casos, una coin-

2 C. Alchourrón y E. Bulygin, "Permission and Permisive Norms", en W. Krawietz et al. (eds.), Theorie der Normen, Berlín, 1984. Ver también P. Navarro y M. C. Redondo, "Permisiones y actitudes normativas", 1988. (Remitido a Doxa.) 
cidencia entre las expectativas de la autoridad normativa y el comportamiento de los sujetos. ${ }^{3}$

b) El comportamiento de los sujetos normativos está sobredeterminado. El conocimiento de la existencia de las prescripciones de $S_{n}$ son solamente un miembro de la disyunción de todas las condiciones suficientes de ciertos comportamientos; o si se prefiere, esta disyunción es un miembro de la conjunción de todas las condiciones necesarias. Tal vez sea este caso el más frecuente en relación con las normas jurídicas. Generalmente se afirma que la conducta conforme a la norma jurídica está determinada por un conjunto de circunstancias o motivos además de la norma jurídica. Sin embargo, el problema es identificar el criterio de pertenencia de las normas jurídicas a la clase de condiciones genéricamente suficientes. Para resolver este punto, podemos utilizar la distinción de Von Wright entre "poder-hacer" de logro y "poder-hacer" de habilidad relativos a los actos de las autoridades normativas. ${ }^{4}$ Esta distinción se vincula con la clásica distinción entre acto-individuo y actocategoría. Cuando hablamos de las normas como condición genéricamente suficiente, las vinculamos con el "poder-hacer" de habilidad, esto es, la habilidad de compelir y evitar. Pero el "poder-hacer" de habilidad implica una clase no vacía de "poder-hacer" de logro. Con esto retornamos al punto de partida. Si el criterio para incluir las normas en la disyunción de todas las condiciones suficientes de una acción es que existe una clase no vacía de actos exitosos de la autoridad normativa destinados a promover conductas, entonces nos enfrentamos con el siguiente dilema: definimos que la existencia de una relación de correspondencia es equivalente a los actos exitosos de la autoridad y con ello cerramos, por definición, la posibilidad de ausencia de motivación de los sistemas para los casos

3 C. Alchourrón y E. Bulygin, "Definiciones y normas", en El lenguaje del derecho. Homenaje a G. Carrib, Abeledo Perrot, 1983, p. 15.

${ }^{4}$ G. von Wright, Norma y acción: una inwestigación lógica, Tecnos, Madrid, 1970, pp. 66-67. 
de existencia de tal relación; o bien diseñamos un conjunto de situaciones sociales en los que se excluyan la existencia de condiciones suficientes no normativas. Sin embargo, este problema - cierre del sistema - no puede ser resuelto de manera definitiva. $^{5}$

2b) La relación de correspondencia entre el sistema normativo y el comportamiento de los sujetos implica que existe el sistema normativo. Por consiguiente, no puede definirse eficacia en términos de correspondencia entre acciones y prescripciones y, simultáneamente, integrar (la eficacia) las condiciones necesarias de la existencia del sistema. ${ }^{6}$ Este problema ofrece también, como corolario, la cuestión de aquellos sistemas normativos que intersectan con la mayor parte de sus elementos. Podemos presentar esto del siguiente modo:

Sea $T^{\prime}$ un período en el cual rivalizan dos sistemas normativos $S_{1}$ y $S_{2}$. Puede ser el caso que $S_{1}$ tenga un alto grado de correspondencia con las conductas del grupo, y que el sistema rival $S_{2}$ también ostente un alto grado de correspondencia. No hay en esta suposición ninguna contradicción. La verdad de los enunciados de correspondencia impide descartar a $S_{1}$ o $S_{2}$; es decir, la prueba de la existencia del sistema jurídico de $T^{\prime}$ es parcialmente independiente de la relación de correspondencia. Nos quedan a mano dos soluciones:

a) El sistema jurídico de $T^{\prime}$ es aquel que motiva realmente el comportamiento de los sujetos. Éste es solamente un criterio provisorio ya que nos permite detenernos en el caso de que $S_{1}$ o $S_{2}$ sean los motivos de la conducta de los sujetos del grupo. Sin embargo, puede que ni $S_{1}$ ni $S_{2}$ sean eficaces en el sentido de motivación. Negar esta posibilidad sería simplemente dogmatismo. Por consiguiente, tendremos que estipular otro criterio:

5 G. von Wright, Explicación y comprensión, Alianza Universidad, Madrid, 1979, pp. 84 y 88.

"R. Caracciolo, “Interpretación de sentido y conocimiento jurídico”, en Revista Latinoamericana de Filosofia, vol. II, n. 2, 1977. 
b) Existe en $S_{1}$ y $S_{2}$ un conjunto de reglas que no pueden dejar de cumplirse sin que el sistema deje de existir como sistema jurídico del grupo. Tal conjunto de reglas son mutuamente exluyentes. Pero, ¿cómo identificar estas reglas? Para ello es necesario un criterio que asigne distinta importancia a las reglas del sistema. Este criterio puede ser interno o externo al grupo social. Un criterio externo es el enunciado por un observador del grupo. Este criterio presenta el problema de que distintos observadores pueden utilizar distintas pautas para asignar importancia a las normas de $S_{1}$ y $S_{2}$. Por lo tanto, el enunciado "Conforme a las pautas $p_{1}, p_{2}, \ldots p_{n}$, el sistema $S_{1}$ es el sistema jurídico de $T^{\prime \prime}$ y el enunciado "Conforme a las pautas $c_{1}, c_{2}, \ldots c_{n}$, el sistema $S_{2}$ es el sistema jurídico de $T^{\prime \prime}$ pueden ser ambos verdaderos. En otras palabras, más sugerentes pero menos exactas, hemos desplazado el problema desde el lenguaje objeto al metalenguaje. ${ }^{7}$

Un criterio interno es el que adopta un grupo social. Pero no hay ninguna necesidad de que los sujetos del grupo tengan tal actitud ante los sistemas jurídicos. Sin embargo, es más interesante el caso en que los sujetos del grupo tienen tal actitud, ya que esto excede la simple relación de correspondencia.

\section{Eficacia y funcionamiento de los sistemas}

Anteriormente señalamos que es usual vincular al concepto de eficacia -entendido como relación de correspondencia - con las condiciones de existencia de los sistemas jurídicos. Mi objetivo ahora es poner de manifiesto la necesidad de utilizar otro concepto de eficacia, vinculado al funcionamiento de los sistemas jurídicos.

El hecho de que las normas motiven conductas es un fenómeno contingente. La representación de la norma puede estar ausente por completo en el momento de ejecutarse la acción

7 R. Caracciolo, "Conocimiento de normas", en Actas del I Congreso Internacional de Filosofia del Derecho, Buenos Aires, 1982. 
del sujeto. El fracaso o éxito del funcionamiento de los sistemas normativos puede medirse en relación con la eficacia de sus normas. Así como el criterio para evaluar el desarrollo de la técnica de motivación indirecta es la coincidencia entre el sujeto que comete el delito con el que sufre el acto coactivo, el criterio para evaluar el funcionamiento del derecho -en tanto que técnica de motivación indirecta - es la incidencia real de la amenaza de castigos y promesas de recompensas en la conducta de los sujetos.

Ahora me detendré en algunas maneras posibles de interacción entre motivos, sujetos y acciones, con el objeto de refinar nuestra percepción sobre la incidencia real de los sistemas normativos. El desafío será invertir nuestros esquemas conceptuales. Ya no veremos al comportamiento de los sujetos sociales a través del prisma de los sistemas normativos (modelo en el cual todas las acciones son iguales en importancia y dignidad por pertenecer al ámbito del deber ser), sino que analizaremos las acciones de los sujetos y, a trasluz, veremos los sistemas normativos.

\section{Sistemas normales y sistemas de baja intensidad}

Los sujetos de los grupos sociales pueden experimentar con mayor o menor intensidad la presión de los instrumentos de control social. El grado de intensidad con que los sujetos experimentan a los sistemas normativos depende de factores complejos y diversos. Sin embargo, cabe investigar las relaciones que existen entre la función específica de los sistemas jurídicos, la intensidad de la presión ejercida por estos sistemas (segán sea su incidencia real en el comportamiento de los sujetos) y la eficacia de las normas.

Sea $Z$ un conjunto de motivos para un conjunto $X$ de sujetos respecto de un conjunto $Y$ de acciones. Cuando estos conjuntos intersectan en al menos la mitad de sus elementos, serán 
denominados "conjuntos normales". Los conjuntos que no son normales son "conjuntos de baja intensidad".

Cuando decimos intersección de conjuntos nos referimos al hecho de que con cada elemento de $Z, X$ y $Y$ podemos formar - los subconjuntos lógicamente posibles de tres elementos (uno de cada conjunto). Si al menos la mitad de los subconjuntos representan relaciones verdaderas, entonces los conjuntos $Z$, $X$ y $Y$ intersectan normalmente.

La determinación de la existencia de un motivo es una cuestión empírica; y como cuestión empírica, podemos fallar - como ocurre a menudo - en determinar si cierto sujeto ejecuta una acción con tal o cual motivo. Pero esto no afecta el problema conceptual. Que existan criterios claros no significa encontrar con facilidad ejemplos claros.

Hay un subconjunto $Z_{1}$ que es de especial interés para nosotros. Es aquel conjunto cuyos elementos son motivos originados en normas jurídicas, en la imputación de una sanción jurídica. Cuando la mayoría de los elementos del conjunto $X$ realizan la mayoría de las acciones que pertenecen a $Y$ en razón de la mayoría de los elementos de $Z_{1}$, entonces diremos que el sistema jurídico que subyace a la conducta del grupo es un sistema jurídico normal. Un sistema normativo es de baja intensidad cuando no es normal. La baja intensidad del sistema normativo puede originarse en $Z, X \circ Y$.

Si los elementos de $X$ o $Y$ intersectan normalmente pero $Z_{1}$ posee baja intensidad, entonces el sistema normativo fracasa en su función de motivar conductas. Obsérvese que los sujetos producen las acciones prescritas por el sistema normativo pero el sistema normativo no es relevante para la decisión de los sujetos. Esto, adviértase, no implica que el sistema normativo no cumpla otra función, ya que puede utilizárselo para la justificación de la crítica por la desviación de las conductas prescritas, constituyendo la base fáctica que subyace a la fundamentación de las sanciones. 
La baja intensidad de $Z_{1}$ convierte al sistema normativo en un sistema de baja intensidad, ya que los sujetos realizan las conductas por motivos diferentes al premio o el castigo. Esto es crucial: si los sistemas normativos funcionan como una técnica indirecta y los sujetos no tienen en cuenta la sanción (en sentido amplio) cuando ejecutan sus acciones, entonces los sistemas normativos no cumplen la función asignada.

Puede suceder que nuestro sistema normativo sea defectuoso porque las acciones calificadas como obligatorias, prohibidas o permitidas no tienen asociada una sanción. No discutiré aquí si estos sistemas son o no son jurídicos, y me limitare a recordar que, para Kelsen, las normas que no prescriben sanciones son normas no independientes, que valen sólo en conexión con aquellos que prescriben actos coactivos. Sin normas que prescriban actos coactivos no estamos habilitados a presuponer la Norma Básica Fundante, y por ende, no hay sistemas jurídicos.

$\mathrm{Al}$ estar ausente la sanción, el conjunto $Z_{1}$ es un conjunto vacío. El sistema normativo no inspira temor ni deseo, es irrelevante como técnica de motivación.

Puede ser el caso que $Z_{1}$ se origine en un conjunto de normas que pertenecen a más de un sistema normativo. Los sistemas normativos no son independientes ya que poseen elementos en común. Supongamos que los sistemas normativos son $A$ y $B$, y que $B$ es más extenso que $A ; A$ está incluido en $B$. Si el conjunto $Z_{1}$ es normal respecto de $A$ puede no serlo respecto de $B$, y por consiguiente el sistema $A$ puede ser normal y $B$ ser un sistema de baja intensidad.

Ejemplo:

$$
A=\left\{N_{1} ; N_{2}\right\} \quad \begin{array}{r}
B=\left\{N_{1} ; N_{2} ; N_{3} ; N_{4} ; N_{5}\right\} \\
Z_{1}=\left\{N_{1} ; N_{2}\right\}
\end{array}
$$

Supongamos que $X$ y $Y$ son conjuntos normales. Nuestros enunciados de eficacia, es decir, enunciados acerca del funcionamiento de los sistema normativos, tendrán valor positivo para el caso del conjunto $A$ y negativo para el caso del conjunto 
$B$. Adviértase que esto no significa que las acciones que pertenecen a $Y$ no sean las mismas que prescribe el conjunto $B$, sino que las normas $N_{3}, N_{4}$, y $N_{5}$ son irrelevantes -dentro del segmento temporal analizado- para las conductas de los sujetos del conjunto $X$. Por lo tanto, la intensidad del funcionamiento del sistema de motivación representado por el conjunto normativo $B$ es escasa. La relación de inclusión tampoco asegura que si el sistema normativo $B$ posee la propiedad de ser normal, el sistema $A$ también lo sea, ya que el sistema $B$ puede ser normal en virtud de que: $Z_{1}=\left\{N_{3} ; N_{4} ; N_{5}\right\}$. Por último, que el conjunto $Z_{1}$ originado en $A$ o $B$ sea normal, no implicará que los sistemas normativos $A$ o $B$ sean normales. La normalidad de $Z_{1}$ es una condición necesaria pero no suficiente de la normalidad del sistema normativo subyacente.

\section{Conclusiones}

- Un concepto de eficacia útil para la teoría general del derecho tiene que relacionarse lógicamente con el concepto de prescripción.

- La vinculación entre eficacia y prescripción no se agota en los enunciados de correspondencia sino que involucran un componente contrafáctico sobre la incidencia de los sistemas normativos.

- El concepto de eficacia vinculado sólo a la relación de correspondencia no da cuenta del carácter normativo de los sistemas jurídicos y no proporciona un criterio sólido de existencia.

- El concepto de eficacia vinculado a los motivos de los sujetos sirve como criterio para evaluar el funcionamiento de los sistemas jurídicos y pone de manifiesto que el problema de la eficacia no es un simple recuento de estados de cosas coincidentes con los contenidos normativos.

- La relación entre motivos y acciones permite reconstruir el 
problema de la eficacia a la luz de una teoría de condiciones necesarias y suficientes.

\section{BIBLIOGRAFIA}

Kelsen, H., Teoría pura del derecho, UNAM, 1979.

Alchourrón, C. y E. Bulygin, "Permission and Permisive Norms" en W. Krawietz et al. (eds.), Theorie der Normen, Berlín, 1984.

Navarro, P. y M. C. Redondo, Permisiones y actitudes normativas, 1988 (remitido a Doxa).

Alchourrón C. y E. Bulygin, “Definiciones y normas”, en El lenguaje del derecho. Homenaje a G. Carrió, Abeledo Perrot, 1983.

G. von Wright, Norma y acción: una investigación lógica, Tecnos, 1970.

, Explicación y comprensión, Alianza Universidad, 1979.

Caracciolo, R., "Interpretación de sentido y conocimiento jurídico" en Revista Latinoamericana de Fülosofia, vol. II, núm. 2, 1977.

, "Conocimiento de normas" en Actas del I Congreso Internacional de Filosofia del Derecho, Buenos Aires, 1982.

Recibido: 24 agosto 1989. 


\section{SUMMARY}

In this paper, I analize the relation between efficacy and legal systems. The legal theory, generally, emphasizes the importance of the efficacy as an existence criterion of legal systems, though, the jurists do not much to clarify the precise meaning of this concept. Nevertheless, we can relate the efficacy with the performance of legal systems. In this way, first, I criticize the concept of efficacy as the mere correspondence between state of affairs and norm contents; and, secondly, I analize the concept of efficacy as the relation between motives, norm subjects, and actions. 